$\mathbb{P}$ periodica polytechnica

Civil Engineering

$52 / 2(2008) 6371$

doi: 10.3311/pp.ci.2008-2.02

web: http://www.pp.bme.hu/ci

(c) Periodica Polytechnica 2008

RESEARCH ARTICLE

\section{Particle tracking velocimetry (PTV) and its application to analyse free surface flows in laboratory scale models}

\author{
Béla Sokoray-Varga / János Józsa
}

Received 2008-08-18, accepted 2008-10-15

\section{Abstract}

The methodology and application of particle tracking velocimetry (PTV) for surface flow velocity measurements are presented in a conventional laboratory scale model characterised both by large vortices and turbulence, making use of the fact that PTV allows to take reliable measurements in flows exposed to strong deformations typical in turbulent shear layers.

The experimental setup included a river reach with main channel and wide floodplain of different roughness. Surface PTV measurements were carried out by a CCD camera with 30 frames per second frame rate and vertical camera axis.

In the evaluation of the recordings an algorithm suitable to be applied in flows with strong strains was needed, capable to find corresponding particles even by highly deformed neighbourhoods. For this aim a modification of the so-called velocity gradient tensor method was used.

The reconstructed velocity fields were used first to visualize the mean flow field, then to identify and investigate the various flow structures by means of the swirling strength analysis.

\section{Keywords}

Particle Tracking Velocimetry (PTV) · PIV · laboratory scale model $\cdot$ surface velocity measurement

\section{Acknowledgement}

We would like to thank the Institute for Hydromechanics at University of Karlsruhe for providing insight into their PIV laboratory works. Special thanks to Wernher Brevis for instructing and explaining algorithmic details of his PTV code, inspiring then the one used in the present work. The research was partly funded by the Hungarian Scholarship Board Office.

\section{Béla Sokoray-Varga}

Department of Hydraulic and Water Resources Engineering, BME, Múegyetem rkp. 3. Budapest, H-1521, Hungary

e-mail: sokoray@vit.bme.hu

\section{János Józsa}

Department of Hydraulic and Water Resources Engineering, BME, Múegyetem rkp. 3. Budapest, H-1521, Hungary

e-mail: jozsa@vit.bme.hu

\section{Introduction}

The developement of Particle Image Velocimetry (PIV) and Particle Tracking Velocimetry (PTV) techniques in the last decades allow their applications in more and more complex environments, e.g. in flows characterised by coherent structures and enhanced turbulence. Their use in laboratory conditions for extracting basic flow features such as the mean (translational) velocity fields or the turbulent kinetic energy field has become widespread.

One of the most fundamental tools for measuring turbulence is the Acoustic Doppler Velocimeter (ADV), as it can detect the 3-dimensional flow velocity of a small fluid volume at high temporal resolutions. The ADV inherently provides Eulerian velocity data of a single point, and its robust version is suitable even for field measurements.

In the contrary, the above mentioned image velocimetry techniques can measure flow velocity fields at temporal resolutions comparable to or even better than the ADVs. If using one single camera, the recorded velocities are two-dimensional, if using two cameras, the recorded velocities are three-dimensional. A clear advantage compared to the ADV technique is that image velocimetry provides flow velocity information of a larger area of the flow simultaneously. These characteristics make image velocimetry suitable for detecting fast space-time changing flow features, thus offering a promising tool to space domain analysis of coherent structures and turbulence.

In our paper we present the methodology and application of PTV for surface flow velocity measurements in a conventional laboratory scale model, characterised both by large vortices and turbulence, making use of the fact that PTV allows to take reliable measurements in flows exposed to strong deformations typical in turbulent shear layers e.g. between the main channel and the floodplain of a river.

The obtained velocity fields are used first to visualize the mean flow field, then to examine the various flow structures by means of swirling strength analysis. 


\section{Overview of PTV and PIV techniques}

PTV and PIV are both image velocimetry techniques, whose common methodological principle is that tracer particles are added to the flow, assumed to move with the local flow velocity, and the flow with the illuminated tracer particles is recorded with cameras. Local flow velocities are reconstructed by determining the displacements of the tracer particles between two subsequent images (called frames) over the time delay between the recording of the frames.

The determination of the displacement is feasible as a particle belongs to a characteristic group (let it be called neighbourhood) which has a specific local distribution pattern. For the comparison of neighbourhoods in the evaluation procedure most algorithms use cross-correlation analysis between a referenced neighbourhood in the first frame and a possible candidate neighbourhood in the second frame.

As PTV volved first form PIV as a special method, the two techniques have several common characteristics, which are well summarized in e.g. [7]. First of all, they provide nonintrusive velocity measurements as they are optical measurement techniques with no need of a probe unavoidably disturbing the flow. Surface velocities and velocities within the water body can also be measured, by illuminating only tracer particles in the plane of our interest within the water body by an appropriate plane light sheet. As light is scattering from the particles in all direction, they become point light sources and thus visible in the image. Plane light sheet illumination can be well achieved by lasers.

Such techniques are whole field techniques as it is possible to record images and provide flow velocity information of large parts of flow fields simultaneously. This allows to study the spatial distribution of the instantaneous velocity field including the detection of spatial structures (even in highly unsteady conditions).

The spatial and temporal resolution of image velocimetry measurements is, however, limited due to technical restrictions. The frame rate (fps) of the camera mainly determines the temporal resolution of the recording, and the resolution (pixel number) of the camera sensor determines the spatial resolution, moreover, the size of the tracers also have an effect on the spatial resolution as will be pointed out later.

The measurement done by these techniques are indirect velocity measurements in the sense that the displacement of the tracer particles in the flow is measured directly whereof the velocity of fluid elements is reconstructed. For this reason, the selection of proper tracer particles requires careful considerations as they ought to move together with the ambient fluid elements faithfully.

If the density of the fluid and that of the tracer particles do not match, gravitational forces will influence the accuracy of the measurements. The size and the shape can also influence the tracers in properly moving with the flow, for example in critical areas like around narrow slots, where streamlines strongly converge, tracers can become too large relative to the local flow scale, thus causing data drop-out.

Another aspect on the size of the tracers is that a tracer needs to have an extension as large as at least $3 \times 3$ pixels in the digital images for good particle detection of the evaluating algorithm, as for sub-pixel accuracy determination of the particle centroid the intensity distribution is needed from peak-neighbour pixels.

For an appropriate spatial resolution of the measurements a compromise has to be found between the camera resolution, the particle size and the area to measure. As mentioned before a tracer has to be at least $3 \times 3$ pixels in extension, but at the same time as the size of the spatial structures to be recorded is not dependent on the tracer's size flew, structures smaller than the tracer size are evidently not recorded, and structures representated by too few tracers only are still poorly detected.

The temporal resolution limits the magnitude of the velocities that are possible to record. At too high velocities the investigated particle may go out of the image, and at high velocity gradients the neighbourhoods may become too much deformed, which would prohibit the recognition of correspondances.

As the velocities are calculated from recorded images, with a single camera only 2 components of the velocity vectors can be measured (the projection of the 3D vector into the recording plane), and the out-of-plane component is not recorded. There exist several methods to get the out-of plane velocity component mostly using multiple-camera systems (e.g. stereoscopic PIV).

The difference between PTV and PIV is the art of the evaluation of the recordings. In PIV all images are subdivided into small regular sub-areas called "interrogation areas", which are evaluated separately from each other. It is assumed that all particles within one interrogation area have moved quite homogeneously between the two recordings, and the displacements of these interrogation areas are determined by cross-correlating the interrogation area in the first frame with the 2-dimensional shiftings of the corresponding interrogation area (the one of the same position) in the second frame. [7]

On the one hand, this means PIV is providing velocity vectors in a regular raster (one vector for each interrogation area), which makes easier the error filtering and the post-processing of the data. On the other hand, a couple of conditions have to be fulfilled during the measurements. For example, the extent of the particle displacements has to be smaller than the interrogation areas for meaningful correlation factors. Another one is that all interrogation areas need to include several particles in order to ensure real neighbourhood-correlations, which requires the particle distribution in the recorded flow to be nearly homogeneous, difficult to satisfy in turbulent shear flows.

To the contrary, in PTV each individual particle is recognized and identified separately, and tried to be rediscovered in the second frame [3]. The displacement of each individual particle is effectively determined using the information of the neighbourhood. This means PTV provides a displacement vector for each particle, which is efficient in terms of processing time at lower particle numbers. 
The vectors obtained by PTV are, however, not regularly placed, so false vectors can be more difficult to eliminate. But the resulting vector fields enable the tracking of particles, which can be then used to calculate e.g. interesting Lagrangian features. In addition, the particles do not have to be homogeneously distributed in case of PTV, and the limit of the extent of displacements is not any more the size of the interrogation area but the degree of the deformations of the flow field, as at too large deformations the neighbourhoods may not be similar any more (which prohibits the correlation).

The measurements analysed in the present paper were surface velocity measurements, where the occurrence of inhomogeneous particle distributions could not be avoided, one of the reasons to use a PTV algorithm, the details of which will be given later in chapter 4 .

\section{Experimental setup and the measurement system}

The investigated free surface flow was a laboratory scale model of a section of river Maros, a medium size river in the south-east of Hungary, including the main channel and the wide flood-plain with different roughness and water depth. The floodplain also contained some areas with roughness further enhanced by floodplain forest, marked with gray (Fig. 1). The scale of the model was 1:350 horizontally and 1:70 vertically. By $8 \mathrm{l} / \mathrm{s}$ discharge at the upstream boundary sections, the water depth was about 10.5 and $2.5 \mathrm{~cm}$ in the main channel and in the floodplains, respectively.

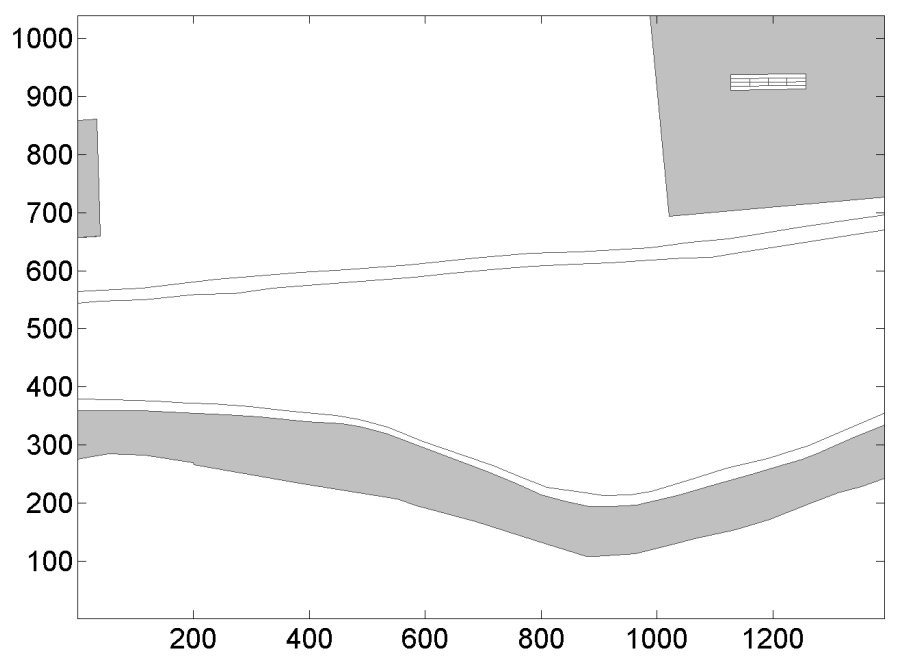

Fig. 1. The experimental setup. (Scale length in the right top corner is 20 $\mathrm{cm})$

The recording device was a monochrome CCD camera with $1392 \times 1040$ pixel resolution and 30 frames per second frame rate. The recordings were made with vertical camera axis (perpendicular to water surface), in order to minimize perspective distortion in the recordings. The distance of the camera lens from the measured water surface was $2.85 \mathrm{~m}$, this enabled to record the flow on an area of about $2 \mathrm{~m} \times 1.5 \mathrm{~m}$.

The monochrome camera provides grayscale images from the flow, which means that a brightness intensity is stored for every pixel. Tracers are distinguished from the background in the evaluation procedure by selecting an appropriate level of brightness as a threshold, above which pixels are considered belonging to a particle image, whereas belonging to the background otherwise. Hence, the quality of a recording is better, if there is a good contrast between the tracer particles and the background for easier distinction, therefore tracer particles with no translucent white colour were selected, refracting more light.

As already emphasized, the selection of the tracer particles needs special care, because PTV measures directly the velocity of the tracers, so if the tracers do not follow the flow faithfully the measurement accuracy will drop significantly, as one of the several sources for such errors.

During our experiments free surface measurements had to be done, therefore tracer particles that remain mostly on the water surface were needed. This can be achieved by using tracers with only somewhat lower density than water density, since choosing too low density thus enabling tracers to significantly emerge from water would lead to measurement errors as well. On the one hand, tracers not having enough drag, may not be drifted sufficiently by the flow, leading to errors mentioned above. On the other hand, it was observed that emergent tracer particles showed unfavourable agglomerating ability, as the surface tension of water around the tracers dragged particles to each other. The liability of particles to form agglomerates is well known in surface measurements, and if the drag force is hampering particles in following the flow faithfully, their motion will not map properly dynamic velocity variations, typical to coherent structures and turbulence, thus becoming useless for the measurement.

We observed that tracers with disc-like shape, and with a density somewhat lower than, but as close as possible to water density are floating on the surface, whereby their upper edge is located just at the free surface level (without emerging from the water body) and have not shown agglomerating feature, but had enough drag to be drifted by even highly curved flow. Such particles, however, are not easy to find. As a result of several tests, we used HDPE curtain rollers with a density of $0.97 \mathrm{~kg} / \mathrm{dm}^{3}$, diameter of $9 \mathrm{~mm}$ (with a of $2 \mathrm{~mm}$ diameter hole in the middle) and a thickness of $3.5 \mathrm{~mm}$. The pixel resolution of the camera would have enabled to record a larger part of the flow, but due to the unavoidable contrast-decreasing effect of the hole in the middle of the tracers the measurement area had to be restricted to the above mentioned size for good quality particle detection.

\section{Description of the applied PTV analysis algorithm}

The PTV analysis consists of a couple of subsequent procedures in order to extract the velocity data from the image sequence. During pre-processing the recorded images are made ready for the analysis, followed then by the particle detection which determines the coordinates of the centroid of every individual tracer particle detected in the images. Finally a parti- 
cle tracking algorithm identifies each particle in the subsequent frame, at the same time determining the displacement in such a way.

\subsection{Image pre-processing}

As an already mentioned important requirement, a good contrast between the tracer particles and the background is essential for good digital evaluation. When recording the flow field with the tracers in the scale model, the background became also visible, because the bottom surface was not black (in fact it was conventional gray concrete), in addition, it was roughened by small stones of very light-gray colour.

The particle detection procedure uses the brightness (intensity level) of the image pixels to find the tracers, therefore stones and other objects of such a brightness would disturb the particle detection. But luckily, using the fact that these stones are steadily present in all frames with no displacement, they can be eliminated during the pre-processing by substracting the background from every recorded image. In order to substract the background, an empty image has to be created that contains no tracers but only the background of the flow field.

An empty background image can be created either by recording a frame without tracers at the beginning of the measurement, or by calculating the mean image from the sequence of the recorded frames, that is calculating the mean values of every separate pixel over the sequence of frames. As to the latter, it has to be taken into account that slowly moving tracer particles contribute more to the pixel brightness along their path on average, and therefore may be eliminated during the substraction.

Once having substracted the mean image from every recorded frame, the substracted frames become nearly black and white containing only some background noise. Note that if the background contains elements with a pixel-brightness near the brightness of the tracers, tracers overlapping with such elements may disappear after the substraction.

\subsection{Particle detection}

The particle detection part of the analysis has to identify every individual particle separately, and has to determine their centroids' coordinates with sub-pixel accuracy.

The particle detection was done using the particle mask correlation method [8], where a typical brightness pattern of a particle image (called particle mask) is generated and every recorded frame is scanned from pixel to pixel to find occurrences of this pattern.

Generally typical particle images in recorded frames can be well described by local two-dimensional Gaussian distribution of the pixel intensity levels (especially in case of particles illuminated by a plane light sheet). [8] proposed therefore to generate the particle mask using such a distribution of the intensities. However, it often occurs that the shape of the particle images becomes elliptical instead of circular due to the inhomogeneous illumination or due to high velocities. In order to support ellipti- cal particle image identification [2] introduced shape modifiers $\mathrm{A}$ and $\mathrm{B}$ to turn the two-dimensional Gaussian distribution elliptical.

In case of our special tracers the maximal brightness (intensity) of the particle image is not located in the central pixel of the particle image (as there is a hole in the middle), therefore the expression had to be completed by a parameter $p$ that shifts the zone of maximal intensities concentrically away from the centroid as follows:

$$
I(x, y)=\exp \left(-\frac{\frac{\left(x-x_{0}\right)^{2}}{A^{2}}+\frac{\left(y-y_{0}\right)^{2}}{B^{2}}-p}{2 \cdot \sigma^{2}}\right)
$$

where $I(x, y)$ is the gray scale intensity at pixel of position $(x, y),\left(x_{0}, y_{0}\right)$ is the object centroid, $\sigma$ is the standard deviation of the Gaussian distribution, $A$ and $B$ are shape modifiers introduced by [2], and $p$ is the distance of the pixels with maximal intensity from the object centroid.

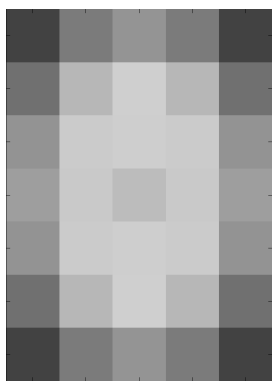

Fig. 2. Generated particle mask

As can be seen in Fig. 2, this modification allowed to generate a particle mask with elliptical shape, whose central pixel intensity is less than the maximal intensity.

To find occurances of this pattern, every recorded frame is scanned from pixel to pixel. This means a frame-segment of the size of the particle mask is selected centered to the actually scanned pixel $\left(x_{0}, y_{0}\right)$, and the two-dimensional crosscorrelation coefficient between the particle mask and the framesegment is calculated as follows:

$$
r\left(x_{0}, y_{0}\right)=\frac{\sum_{m} \sum_{n}\left(P_{m n}-\bar{P}\right)\left(I_{m n}-\bar{I}\right)}{\sqrt{\left(\sum_{m} \sum_{n}\left(P_{m n}-\bar{P}\right)^{2}\right)\left(\sum_{m} \sum_{n}\left(I_{m n}-\bar{I}\right)^{2}\right)}}
$$

where $r\left(x_{0}, y_{0}\right)$ is the cross-correlation coefficient at frame coordinates $\left(x_{0}, y_{0}\right), P_{m n}$ is the intensity of the particle mask in position $(m, n), I_{m n}$ is the intensity of the frame segment in position $(m, n)$

As a result, each pixel of the frame receives a correlation coefficient between -1 and 1 , representing the level of similarity to the particle mask. This is called the correlation map. In principle the pixels with correlation peaks (highest correlation coefficient values) can be considered as central pixels of the particle images. Correlation peaks located nearer than the radius of 

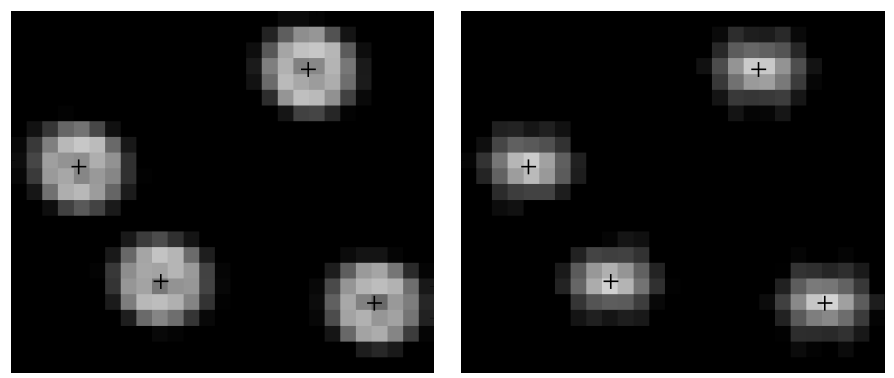

Fig. 3. Detected particle centroid locations on the recorded image and on the correlation map

the tracer particle are considered belonging to the same particle, assuming in case of free surface measurements particle image overlapping hardly occurs.

In case of recorded frames with background noise, if the local distribution of the noise has a brightness pattern similar to the particle mask (even if at very low intensities), the correlation coefficient will give relatively high values, which results in false particle detection. To avoid such errors, the pixels to be masked have to be selected from the frames before executing the particle mask correlation process, for example by selecting all pixels above an threshold intensity level. (This was the reason to achieve good contrast of the recordings by the mean image substraction).

To increase the accuracy of the particle position determination beyond the resolution given by the pixel number of the CCD sensor, the particle centroid locations have to be estimated with sub-pixel accuracy. An interpolation method has to be used that minimizes the so-called "pixel-locking" effect in the estimation, which means that the estimated centroid location does not remain close to the integer-pixel position.

In case of recordings, where the maximal brightness of a particle image is at the central pixel, the intensity distribution of the nearby pixels can be used for the sub-pixel estimation. Assuming that the particle image has a Gaussian distribution [11] used 3 point Gaussian interpolation to estimate the position of the centroid. In fact, they used two one-dimensional Gaussian curves (in orthogonal directions) by using 2-2 neighbour pixels in both directions. The particle centroid location is then estimated to be at the centroid of the interpolated curves. The required correction of the particle centroid location is calculated in $X$ direction as:

$$
\Delta x=\frac{\ln \left(I_{X_{0}-1}\right)-\ln \left(I_{X_{0}+1}\right)}{2\left[\ln \left(I_{X_{0}+1}\right)-2 \ln \left(I_{X_{0}}\right)+\ln \left(I_{X_{0}-1}\right)\right]}
$$

where $I_{X_{0}}$ is the particle image intensity value at the correlation peak pixel, $I_{X_{0}-1}$ and $I_{X_{0}+1}$ are the neighbours. The centroid of the interpolated curve is located at $\Delta x$ with respect to the pixel index $X_{0}$.

As mentioned before, our tracer particles do not induce the maximal brightness (intensity) in the center of the particle image, so Gaussian interpolation can not be applied for the particle images. In turn, as presented in Fig. 3 the correlation map proved suitable to be used for the Gaussian interpolation, as the distribution of the correlation coefficients shows a Gaussian-like distribution.

Note that [6] improved the Gaussian interpolation by means of using $2 \times 3$ pixels by a two-dimensional Gaussian regression which is using all the 8 neighbour pixels, but in our case it produced some false particle centroids.

\subsection{Particle displacement determination}

The particle tracking algorithm investigates each detected particle in frame 1 and tries to rediscover each one in the subsequent frame2. For finding the corresponding particle in frame2 the local distribution pattern of the neighbour particles (neighbourhood) is mostly used.

There are several algorithms available for PTV, but most of them do not tolerate strong deformations of the flow field. We needed an algorithm that is suitable to be applied in turbulent flows with strong deformations, which means an algorithm capable to find corresponding particles even by deformated neighbourhoods. We finally used a modification of the velocity gradient tensor (VGT) method introduced by [4], presented below. The additional advantage of the method is that it does not use the images of the frames but solely the particle coordinates, whereby a lot of memory and processing time can be saved.

A fundamental procedure in PTV is the comparison of neighbourhoods. The definition of a neighbourhood occurs by selecting the $\mathrm{n}$ nearest neighbour particles of an investigated particle. The example in Fig. 4 shows 5 neighbours (a,b,c,d,e) and the investigated particle (p).

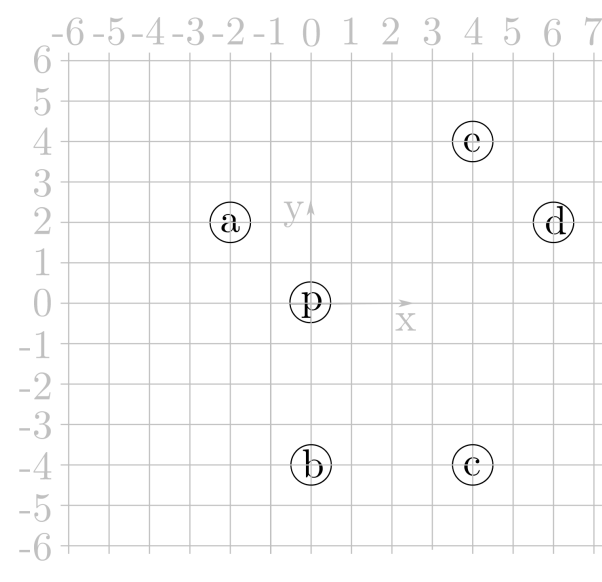

Fig. 4. Neighbourhood around a particle

For comparison of two neighbourhoods, the level of similarity is not expressed by cross-correlation calculations, but by applying the least-squares method as follows:

$$
r=\sum_{i} r_{i}^{2}
$$

where $\left(r_{i}\right)$ is the distance of a neighbour to the nearest neighbour particle, $r$ is considered as the distribution error between two neighbourhoods. The lower this factor, the more similar the neighbourhoods. As an example, the distribution error calcu- 
lated for two neighbourhoods shown in Fig. 5 is:

$$
r=\left(0.5^{2}+1^{2}+1^{2}+0.5^{2}+1^{2}\right)
$$

Another fundamental procedure of PTV is to find candidates from frame 2 that can correspond to a referenced particle in frame1. This is done by looking for particles in frame 2 that are close to the referenced particle's coordinates in frame1. The distance of investigation is a user-defined input parameter that has to be chosen in terms of the extent of the displacement.

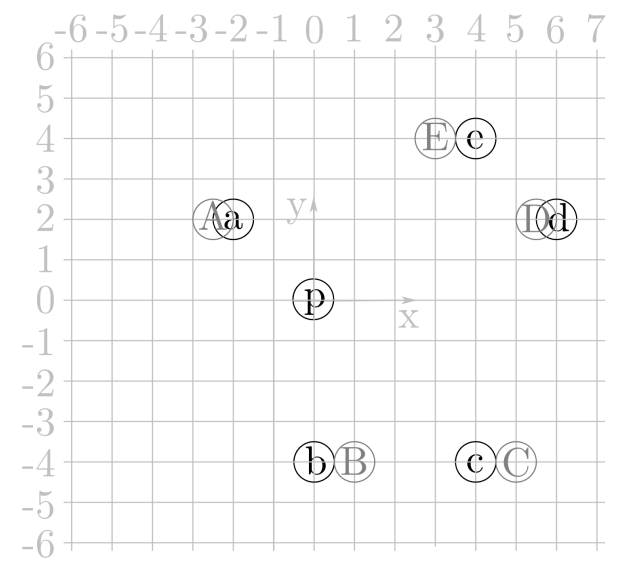

Fig. 5. Comparison of neighbourhoods. Frame1 - black, frame2 - gray

A candidate corresponding to the referenced particle is found by comparing all candidates' neighbourhoods with the referenced neighbourhood by using the least squares method descibed earlier. In order to support strong deformations of the flow field the method applies several deformations on the referenced neighbourhood before comparing it with a candidate neighbourhood.

Applying a particular deformation on the referenced neighbourhood is carried out by multiplying the neighbours' coordinates by a deformation gradient, called velocity gradients by [4]. For example the gradient of the evidently visible deformation of the frames presented in Fig. 5 can be described by -0.5 $\partial x / \partial y$ and $0 \partial y / \partial x$. This deformation gradients could be obtained from the presented neighbourhoods by:

$$
\frac{\partial x}{\partial y}=\frac{x_{e}-x_{E}}{y_{e}}
$$

where $x_{e}, x_{E}$ and $y_{e}$ are the coordinates of the particles $e$ and $E$, respectively.

The deformation gradients to use in the comparison of the referenced and a candidate neighbourhoods can be defined by calculating the deformations using all pairs from the referenced neighbours and the candidate's neighbours. It means the number of examined deformations will be $n^{2}$. The deformations defined this way include the occured deformation several times, therefore the disapperace of a neighbour does not influence the efficiency of the particle tracking.

The lowest achievable distribution error $r$ is kept for every candidate, and finally the candidate providing the lowest one is considered as the corresponding candidate of the referenced particle.

Note that these gradients support only linear deformations in principle, but due to the least squared errors method not only perfectly overlapping neighbourhoods will be recognized as corresponding ones, and the method may remain efficient in case of stronger deformations as well.

\subsection{Error filtering}

By disapperace of particles or too strong deformations not supported by the particle tracking algorithm, some erroneous vectors always show up in the analysis. Most of them point to destination particles that already have valid vectors pointing onto then. Such erroneous vectors can mainly be filtered out by keeping the vector provided by lower distribution error $r$.

The rest of the erroneous vectors point to tracers not yet taking part in defining a vector. Although such vectors could be well recognized visually as outliers in our experience, the algorithmical filtering of such vectors is quite difficult.

Most filtering methods compare an investigated vector with the neighbouring vectors in the investigated frame and eliminate it if found too much different from the neighbourhood. Hence, such methods are based on local similarity of the flow field, but our experience showed that such algorithms eliminated also valid vectors in the filtering processes (especially in turbulent shear layers), which could then lead to losing valuable information. For this reason we tried to find other ways for filtering outliers.

Instead of studying the local similarity of the velocity vectors in an investigated frame, we focused on the temporal similarity of the velocity vectors in subsequent frames. An examined vector of a particle in an investigated frame was compared to the vector of the same particle in the previous frame, that is in the previous timestep. It is known that acceleration of the particles is limited due to physical rules, that is why the similarity of vectors in time is quite high, thus the filtering can be done by defining a threshold velocity difference.

As in case of free surface measurements the particles are not likely to leave the measurement plane, such pathline investigations proved to be quite efficient in our experiments, in which less than $1 \%$ of the vectors were eliminated, only.

In recordings where particles are much more likely to leave the measurement plane due to the out of plane velocity component, other filtering methods can be used. One of the most widely used algorithms is the median test [9, 10].

\subsection{Post-processing}

PTV provides velocity vectors for particles which are not regularly distributed, hence scattered velocity vectors are obtained for every frame. However, in a number of investigations velocity vectors in a regular raster are needed or at least preferred, therefore the scattered velocities had to be interpolated to a regular grid in each frame. 
The interpolation was carried out by Delaunnay triangulation of the scattered data and by fitting cubic interpolation function, whereby values at data points remained invariant. In our particular case a grid of 70x48 nodes was generated.

\section{Results}

Using PTV, a velocity vector is provided for each individual tracer particle. As was mentioned above, the occurence of inhomogeneous particle distribution is not avoidable in surface measurements, that is why the distribution of the velocity vectors provided by the particle tracking algorithm is inhomogeneous, too.

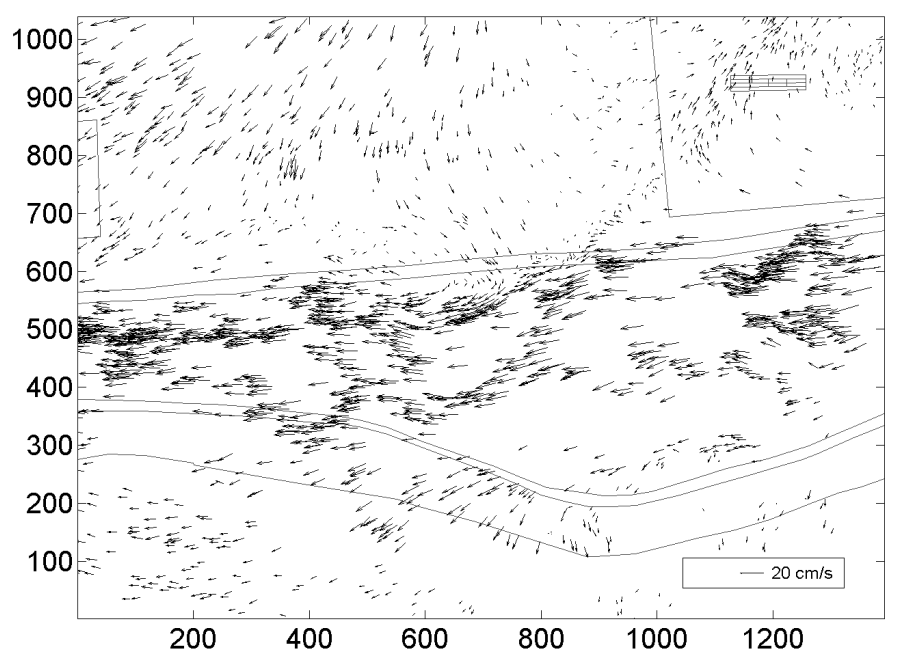

Fig. 6. The scattered velocity vectors provided by the individual particles

It can be seen in Fig. 6, that the distribution of the calculated velocity vectors is inhomogeneous just as the distribution of the particles. As can be also seen, the velocity magnitudes are different in the main river channel and the floodplain, further enhancing irregularity.

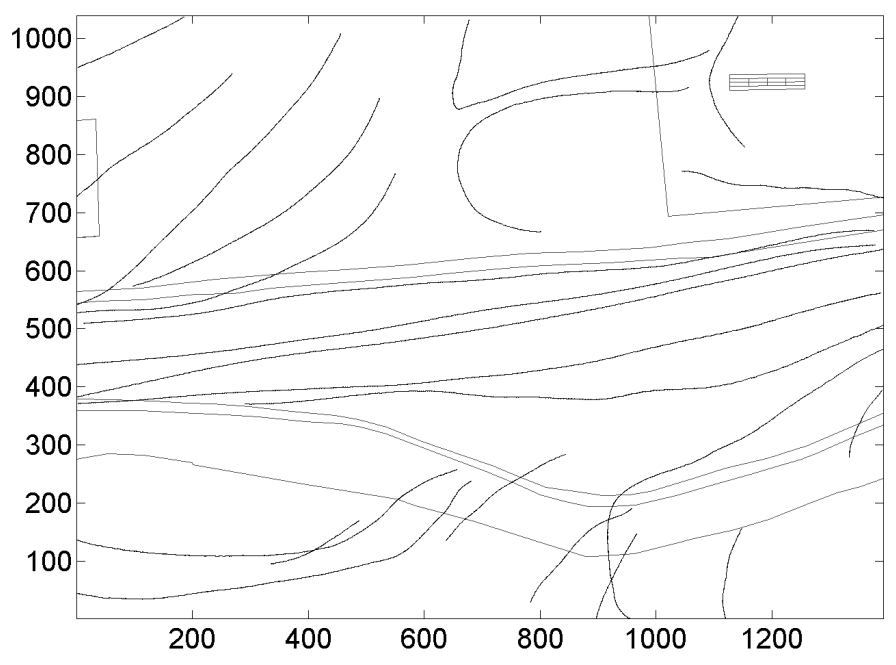

Fig. 7. Some typical reconstructed particle pathlines

As the displacement of every particle is determined in each frame, it makes possible to reconstruct particle pathlines. In our laboratory case particles stayed generally quite long in the surface measurement frame, resulting in rather long pathlines, as seen in Fig. 7. In contrast, in measurements within the water body where usually particles leave the measurement plane (that is the laser light sheet) fast even at very low velocities, the observable pathlines are usually short. Note that in the figure the start of tracking (that is the initial frame) of the particles were not the same, furthermore, the flow was strongly unsteady, all that producing pathlines crossing each other at places.

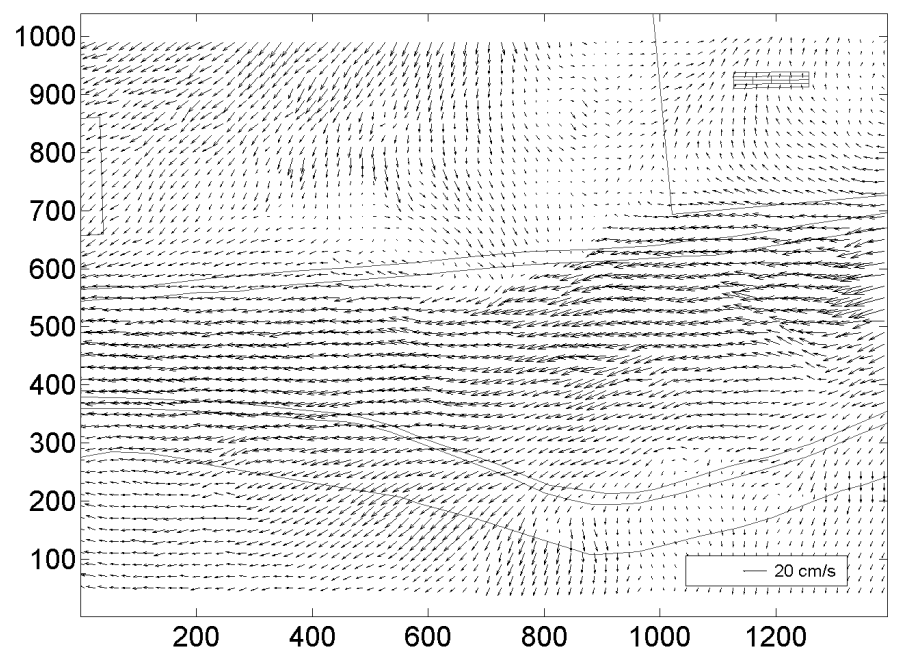

Fig. 8. The velocities interpolated onto a regular raster
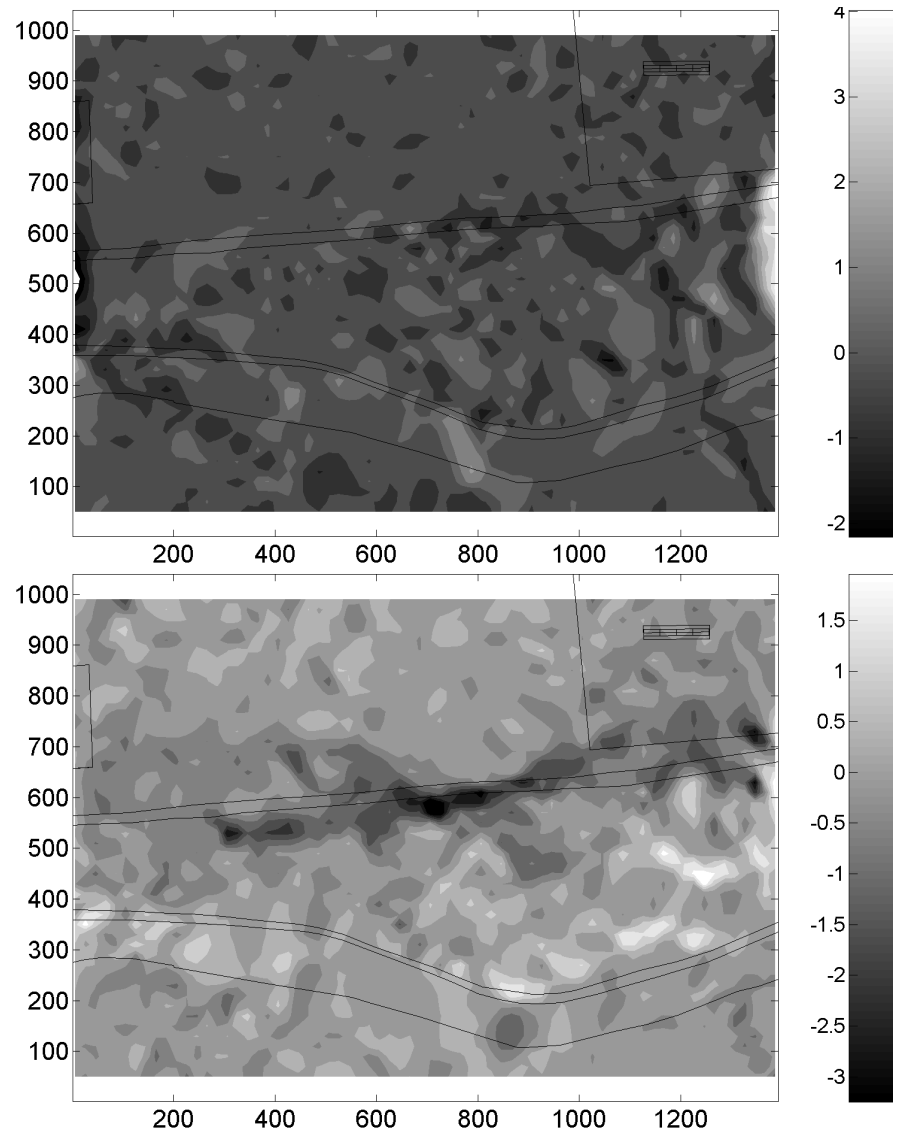

Fig. 9. Calculated divergence and rotation fields for a frame 


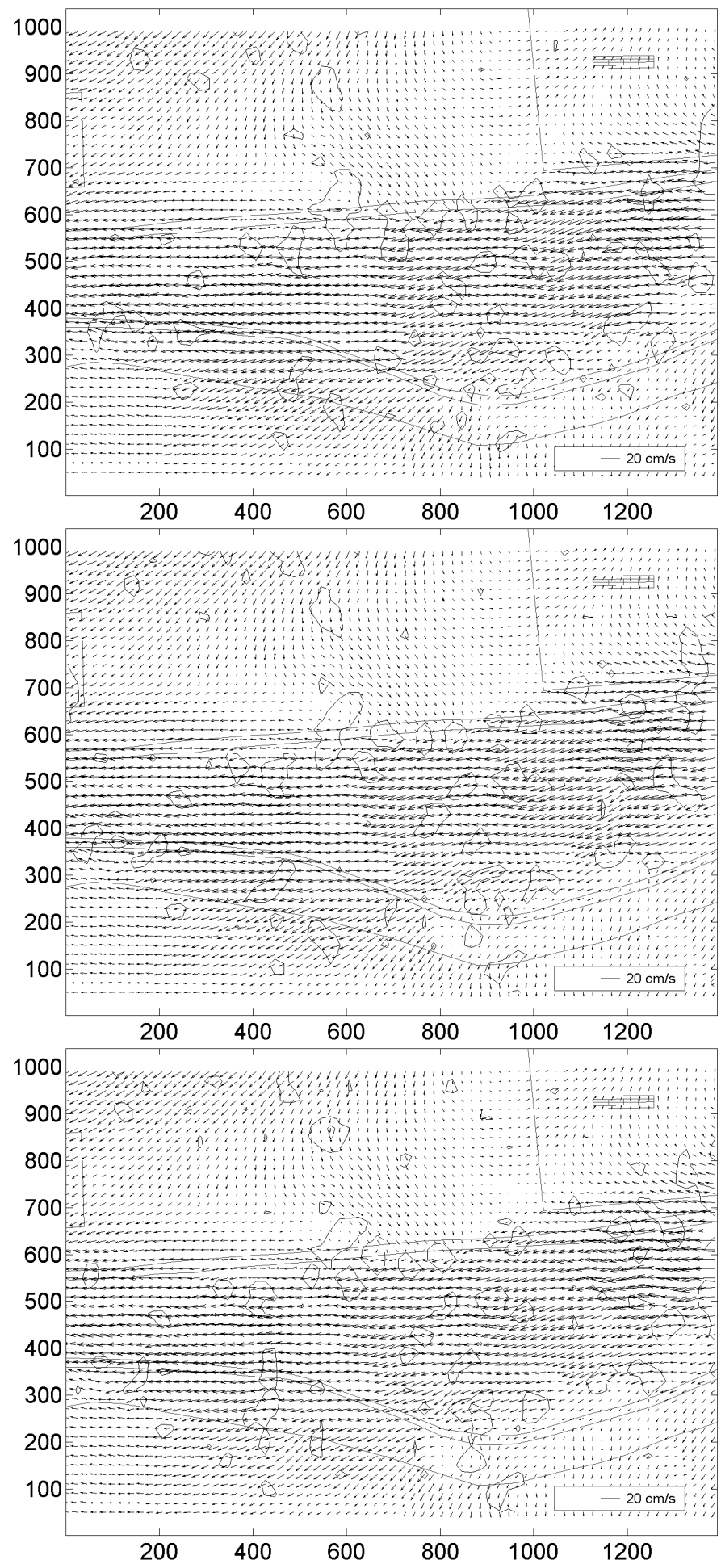

Fig. 10. Vortex identification based on swirling strength analysis. The timestep between the frames is 0.33 seconds

A velocity field interpolated to a regular grid is shown in Fig. 8. The interpolation of the scattered velocity fields facilitates further investigations such as the calculation of the rotation and divergence fields (Fig. 9), time-series analysis, or the detection of coherent structures. As to the rotation and divergence fields, they provide primary information on the surface vortical structures and the up- as well as downwelling zones, respectively.
The two-dimensional velocity vector fields obtained allow to extract even more information for the identification of coherent vortical structures. By comparing several methods, [1] proposed to use the so-called swirling strength analysis for vortex identification based on the eigenvalues of the two-dimensional form of the local velocity gradient tensor $\mathbf{D}^{2 D}$.

$$
\mathbf{D}^{2 D}=\left[\begin{array}{ll}
\frac{\partial u}{\partial x} & \frac{\partial u}{\partial y} \\
\frac{\partial v}{\partial x} & \frac{\partial v}{\partial y}
\end{array}\right]
$$

where $(x, y)$ and $(u, v)$ are are orthogonal directions and velocity components, respectively.

The velocity gradient tensor for two-dimensional velocity fields will either have two real eigenvalues $\left(\lambda_{r 1}, \lambda_{r 2}\right)$ or a pair of complex conjugates $\left(\lambda_{c r} \pm \lambda_{c i}\right)$. The strength of the local swirling quantified by $\lambda_{c i}$ can be understood as the swirling strength of the vortex. Vortices can be identified then by plotting iso-lines and regions of $\lambda_{c i}>0$.

Fig. 10 shows 3 subsequent frames with a timestep of 0.33 seconds with vortices identified by the swirling strength analysis. The effects of the shear layer between the floodplain and the main channel can be seen around $(x=600 . .800, y=600)$, where a series of vortices is travelling along the right bank of the main channel. The subsequent frames allow us to observe the surface fingerprint of those vortices moving more or less at the local translational velocity.

\section{Discussion and conclusions}

The methodology of Particle Tracking Velocimetry and its sample application in a laboratory fluvial scale model have been presented. The surface flow velocity fields were reconstructed by using the modified Velocity Gradient Tensor method, supporting measurements even with strong shear and vorticity.

The agglomeration of the tracer particles could be avoided by choosing appropriate particle density and disc-like shape.

The obtained velocity fields proved suitable for extracting information on coherent vortical structures, even visualising and to some extent quantifying their motion and deformation in the translational velocity field.

The use of PTV techniques has been so far limited mainly to laboratory environment, due to the special requirements for the measurements like transparency of the water body (in case of measuring within the water body) or moderate deformations of the flow field. For measuring surface velocities of the flow, however, this method may be suitable also for field applications in the future.

Last, as the spatial resolution and accuracy of the reconstructed velocity field strongly depends on the tracer distribution density, grids with adaptive refinement capability to the latter (like quad-tree based rastering, see e.g. in [5]) could be efficiently implemented. 


\section{References}

1 Adrian RJ, Christensen KT, Liu ZC, Analysis and interpretation of instantaneous turbulent velocity fields, Experiments in Fluids 29 (2000), 275-290, DOI 10.1007/s003489900087.

2 Estrada CEP, Analysis, comparison and modification of various particle image velocimetry (PIV) algorithms, 2004. Master's thesis.

3 Hassan Y, Blanchat TChS, PIV flow visualization using particle tracking techniques, Measurement Science Technology 3 (1992), 633-642, DOI 10.1016/j.nucengdes.2008.01.027.

4 Ishikawa M, Murai Y, Wada A, Iguchi M, Okamoto K, Yamamoto F, A novel algorithm for particle tracking velocimetry using the velocity gradient tensor, Exp Fluids 29 (2000), 519-531, DOI 10.1007/s003480000120.

5 Krámer T, Józsa J, An adaptively refined, finite-volume model of windinduced currents in lake Neusiedl, Per. Pol. Civil Eng. 49 (2005), no. 2, 111 136.

6 Nobach H, Honkanen M, Two-dimensional gaussian regression for subpixel displacement estimation in particle image velocimetry or particle position estimation in particle tracking velocimetry, Experiments in Fluids 38 (2005), 511-515, DOI 10.1007/s00348-005-0942-3.

7 Raffel M, Willert CE, Kompenhaus J, Particle Image Velocimetry A Practical Guide, Springer, 1998.

$8 \mathbf{K}$, Etoh T, A study on particle identification in PIV - particle mask correlation method, Journal of Visualization 1 (1999), 313-323.

9 Westerweel $\mathbf{J}$, Efficient detection of spurious vectors in particle image velocimetry data sets, Experiments in Fluids 16 (1994), 236-247, DOI 10.1007/BF00206543.

10 Westerweel J, Scarano F, Universal outlier detection for PIV data, Experiments in Fluids 39 (2005), 1096-1100, DOI 10.1007/s00348-005-0016-6.

11 Willert C, Gharib M, Digital particle image velocimetry, Experiments in Fluids 10 (1991), 181-193. 